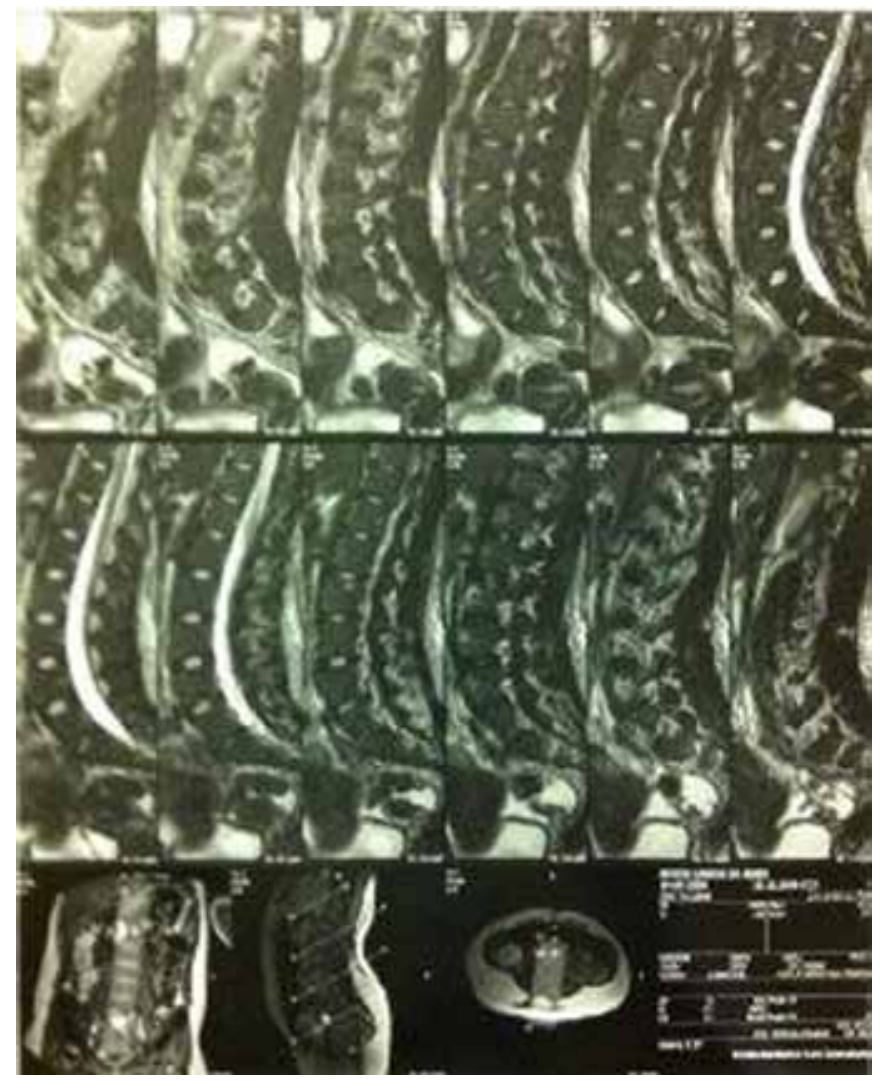

Abstract 563 Figure 1 NMR

Results First treatment was initiated with 2 immunoglobulin's cycles and pulse therapy with methylprednisolone not getting satisfactory motor response. The patient developed with respiratory effort and hypoventilation (PCO2 $\mathrm{max}-90 \mathrm{mmHg}$ ) becoming necessary to introduce a course of 5 days of non invasive ventilation. Subsequently, a 5-day plasmapheresis cycle was iniciated, solving the clinical case with an increasing recovering of motor and ventilation function after 1 week of treatment.

Conclusion Enterovirus infections can cause several clinical manifestations such as transverse myelitis. In most of tranverse myelitis cases the treatment is immunoglobulin and pulse therapy with methylprednisolone, yet this case was only solved after plasmapheresis cycle.

\section{AUTOIMMUNE ENCEPHALOPATHY NINE YEAR OLD BOY WITH NO HISTORY OF INTEREST}

doi:10.1136/archdischild-2012-302724.0564

C Perez Moreno, MDM Campillo. Paediatric Area, Health Centre, Altea, Spain

Background and Aims Nine year old boy admitted with meningeal syndrome. six days ago began with fever being treated with azithromycin. three days before entering a high fever, bilious vomiting, no diarrhea, the next day associated dizziness, headache, drowsiness, the day before admission diplopia and pain associated neck.

Strikes disease, no rashes or petechiae.

Methods Hemogram: 13.24 leukocytes, neutrophils 87.4\%

Cerebrospinal fluid red blood cells $160 / \mathrm{mmc} 30 / \mathrm{mmc}$ polynuclear leukocytes $5 \%$, lymphocytes $95 \%$, Gram negative, PCR Herpes simplex I and II negative, negative enterovirus, varicella zoster negative. Negative blood cultures, Mantoux negative.

Abnormal EEG tracing during wakefulness slow waves of high amplitude delta, acute, which are located in anterior and temporal area.
RMN ill-defined hyperintense areas in pons, cerebellum, basal ganglia, right parietal subcortical white matter. suspected encephalitis retrovirus by areas of parenchymal signal alteration above and infratentorial level.

Three days later reduction in the number and extent of intraaxial lesions above and infratentorial.

Results The clinical and resonancia were doing suspect herpes virus encephalopathy, so income at the start of treatment with intravenous acyclovir. When we receive negative results and the improvement of symptoms, treatment it was suspended treatment with acyclovir on the fifteenth day and start treating autoimmune encephalitis with five boluses of methylprednisolone one gram every 24 hours

Conclusions The day of discharge was treated with $60 \mathrm{mg}$ of prednisolone daily. the fever subsides completely within three days before discharge, and intention tremor persists discrete gait instability.

\section{REFLECTION ON A CASE OF DOPAMINE-RESPONSIVE DYSTONIA}

doi:10.1136/archdischild-2012-302724.0565

ML Neamtu, L Dobrota. Pediatric Clinic Hospital Sibiu, Lucian Blaga University of Sibiu, Sibiu, Romania

Background Characteristic symptoms of Dopamine-responsive dystonia (DRD) are increased muscle tone and Parkinsonian features. Children with DRD are often misdiagnosed. The disorder responds well to treatment with Levodopa.

Aim To reflect on a case of DRD.

Method Case report of a 13 years old girl misdiagnosed with tetany.

Results The girl was hospitalized for opisthotonus, positive Trousseau and Chvostek signs, diagnosed as tetany. The laboratory analysis have shown: normal serum of calcium $(2.28 \mathrm{mmol} / \mathrm{l})$, normal serum of magnesium $(0.80 \mathrm{mmol} / \mathrm{l})$, normal serum of phosphor $(1.26 \mathrm{mmol} / \mathrm{l})$, normal alkaline phosphatase $(261 \mathrm{u} / \mathrm{l})$ and normal PTH $(27.9 \mathrm{pg} / \mathrm{ml})$. Although the initial evolution was favorable (with intravenous calcium gluconate), the hypoparathyroidism diagnosis requiring reconsideration. The final diagnosis was DRD with long good evolution after Levodopa treatment. Referring to family history we learned that the patient have a cousin with the same symptoms.

Conclusions The misdiagnosis results from the following similarities: increased muscle tone with opisthotonus, writer's cramp with Trousseau sign, facial dystonia with Chvostek sign and difficult speech (due to facial dystonia) with patient illiteracy. All these similarities delayed the DRD diagnosis.

\section{KLIPPEL TREUNANAY SYNDROME IN DIFFERENTIAL DIAGNOSIS OF CEREBRAL PALSY}

doi:10.1136/archdischild-2012-302724.0566

'P Karakaya, 'Y Topçu, 'E Bayram, 'U Yiş, ${ }^{2} \mathrm{H}$ Çakmakçı, 'S Hız. 'Pediatric Neurology, ${ }^{2}$ Radiology, Dokuz Eylül University, Izmir, Turkey

In cerebral palsy (CP) atrophy of the paretic body half results in disturbed growth. Disturbed growth is also a feature of a rare disorder; Klippel Treunanay syndrome (KTS). Here we report a child with an initial diagnosis of CP because of limping and thinning of the extremities on the right side who had a final diagnosis of KTS. Five year old male was admitted to our department of Pediatric Neurology. He had been followed up with the diagnosis of CP since he started walking because of limping and thinning of the extremities on the right side of his body. His perinatal and natal period was uneventful. Developmental milestones were normal. On physical examination hypertrophy of the left upper and lower extremities 